\title{
Absorber height effects on SWA restrictions and 'Shadow' LER
}

\author{
Brittany M. McClinton, ${ }^{1}$ Patrick P. Naulleau ${ }^{2}$ and Thomas Wallow ${ }^{3}$ \\ ${ }^{1}$ Dept. of Electrical Engineering, University of California, Berkeley, CA 94720 \\ ${ }^{2}$ Center for X-Ray Optics, Lawrence Berkeley National Laboratory, Berkeley, CA 94720 \\ ${ }^{3}$ GLOBALFOUNDRIES Inc., Sunnyvale, CA 94088
}

\begin{abstract}
In this study, we look at the 3D effects of absorber height on mask patterns for extreme-ultraviolet lithography (EUVL). Our first consideration is the extent to which sidewall angle (SWA) constrains the process window. Taking $10 \%$ of the total CD error budget as an acceptable tolerance, this amounted to $0.2 \mathrm{~nm}$ of tolerable SWA-induced CD error. Results for the three nominal SWA cases show that the angle needs to be constrained to within about 0.5 degrees. Overall, the results above indicate that not only is there not much change in sensitivity on changing angle as a function of nominal angle, but also that the nominal angle has no large effect on process window size. Secondly, we consider how off-axis illumination shadowing of the mask absorber pattern effects line-edge-roughness (LER). Data suggests shadowing causes minimal differences between the left- and right-side LER for the 22nm half-pitch node under disk $\sigma=0.5$ illumination and 70nm absorber height. For 16nm half-pitch with crosspole $\sigma=0.2$, significant differences were seen.
\end{abstract}

Keywords: extreme ultraviolet, lithography, sidewall angle, reflective mask, absorber height effect, shadow effect, lineedge roughness

\section{INTRODUCTION}

As extreme-ultraviolet lithography (EUVL) readies for introduction with the 22-nm half-pitch node or below, several key aspects of absorber height effects remain unexplored. Although investigations into SWA effects such as shifts in the aerial image [1-2] and CD error [3-5] have been made, explicit sidewall angle (SWA) restrictions based on the height of the mask absorber have not yet been clearly defined. In particular, there is not much understanding of how nominal SWA may affect the process window size or sensitivity to changing angle. We make an initial investigation into how tight SWA constraints will need to be based on $10 \%$ of the total CD error budget (which is itself nominally $10 \%$ of CD). Thus, we allot the SWA induced CD error budget a $\pm 0.2 \mathrm{~nm}$ tolerance for $22 \mathrm{~nm}$ half-pitch.

Another absorber height effect that remains little understood is how shadowing alters line-edge roughness (LER). Due to the high absorptivity of materials at EUV wavelengths, EUVL uses an off-axis illumination system on a reflective mask that introduces potential shadowing effects. One potential pitfall that must be carefully monitored is the effect of mask absorber height blocking light from reaching, and therefore, correctly detecting, the base edge position of a feature. While mask features can correctly compensate sizing to target at the wafer, the effects of this shadowing on LER have not yet been investigated. Specifically, 'shadow' LER may exacerbate or mitigate the inherent LER on the mask as it transfers to the wafer. Shadowing may also cause differences in the observed LER on the right and left side of the features. In this paper, we look at shadow LER versus non-shadow LER through focus, for two sample amplitudes for a wide range of spatial frequencies at both the 22-nm and 16-nm half-pitch nodes.

\section{SIDEWALL ANGLE SIMULATIONS}

In our examination of 3D mask absorber effects, we first consider to what extent the sidewall angle is constrained by $\mathrm{CD}$ tolerance. We conduct FDTD aerial image modeling using Hyperlith [6] by Panoramic technology for an aberration-free optical system of $\mathrm{NA}=0.32$, illumination wavelength of $13.4 \mathrm{~nm}, 4 \mathrm{X}$ demagnification, disk illumination with partial coherence factor $\sigma=0.50$ with an angle of incidence of 6 degrees first for illumination perpendicular to the lines and spaces on our reflective mask ( $\phi=0^{\circ}$ so shadowing occurs). The source was amply sampled with 50 source points on the half-axis. The TaN mask absorber was designed at $70 \mathrm{~nm}$ thickness, above a $2.5 \mathrm{~nm}$ Ru capped 40 bilayer multilayer mirror using Fourier boundary conditions (FBC) with a fixed reflectivity curve calculated using optical constants [7-8]. The mask object was designed for measuring $88 \mathrm{~nm}$ feature size at the top surface of the mask feature targeted to $22 \mathrm{~nm}$, and depending on the sidewall angle, was properly biased since Hyperlith measures the feature at the base in wafer 
dimensions (see Fig. 1). Thus, for the three sidewall angle settings we investigated which were 80,85 , and 88 degrees, the CD bias at wafer level was respectively 6,3 , and $1 \mathrm{~nm}$. In each case, we calculated the nominal intensity threshold for correct sizing to target $\mathrm{CD}$ as well as adjusted the defocus offset so that best focus of the bossing curves resided at a nominal zero defocus value.

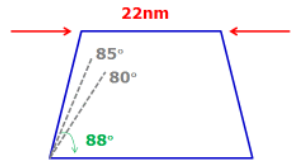

Fig. 1. Nominal SWA was varied from $88^{\circ}$, to $85^{\circ}$, to $80^{\circ}$. The mask was designed to measure $88 \mathrm{~nm}$ at the top of the feature, and depending on nominal SWA, was properly biased to give the target $22 \mathrm{~nm}$ at the base of the feature.

First, we looked at the contrast through focus for the nominal SWA case to determine the range of focus over which the aerial image delivered a contrast above 50\% (see Fig. 2). The 50\% contrast threshold value was arbitrarily chosen as a metric for usable depth of focus over which the feature would print in a standard grade photoresist. The usable depth of focus in all three sidewall angle cases was determined to identically be $\pm 78 \mathrm{~nm}$. We then calculated the optimized process window for this fixed depth of focus range, a full 156nm in all three sidewall angle cases (Fig. 3). Specifically, for each sidewall angle case, we adjusted the intensity threshold to optimize the process window from the nominal intensity threshold value found before when correctly sizing the target $\mathrm{CD}$ at the wafer. Lastly, we took the resulting aerial images based on this optimized intensity threshold for a range of sidewall angles through focus to determine what range of angles still yielded an acceptable CD. Taking $10 \%$ of the total CD error budget as an acceptable tolerance, this amounted to $0.2 \mathrm{~nm}$ of tolerable SWA-induced CD error. Results for the three nominal SWA cases show that the angle needs to be constrained to within about 0.5 degrees (Fig. 4). Overall, the results above indicate that not only is there not much change in sensitivity on changing angle as a function of nominal angle, but also that the nominal angle has no large effect on process window size.

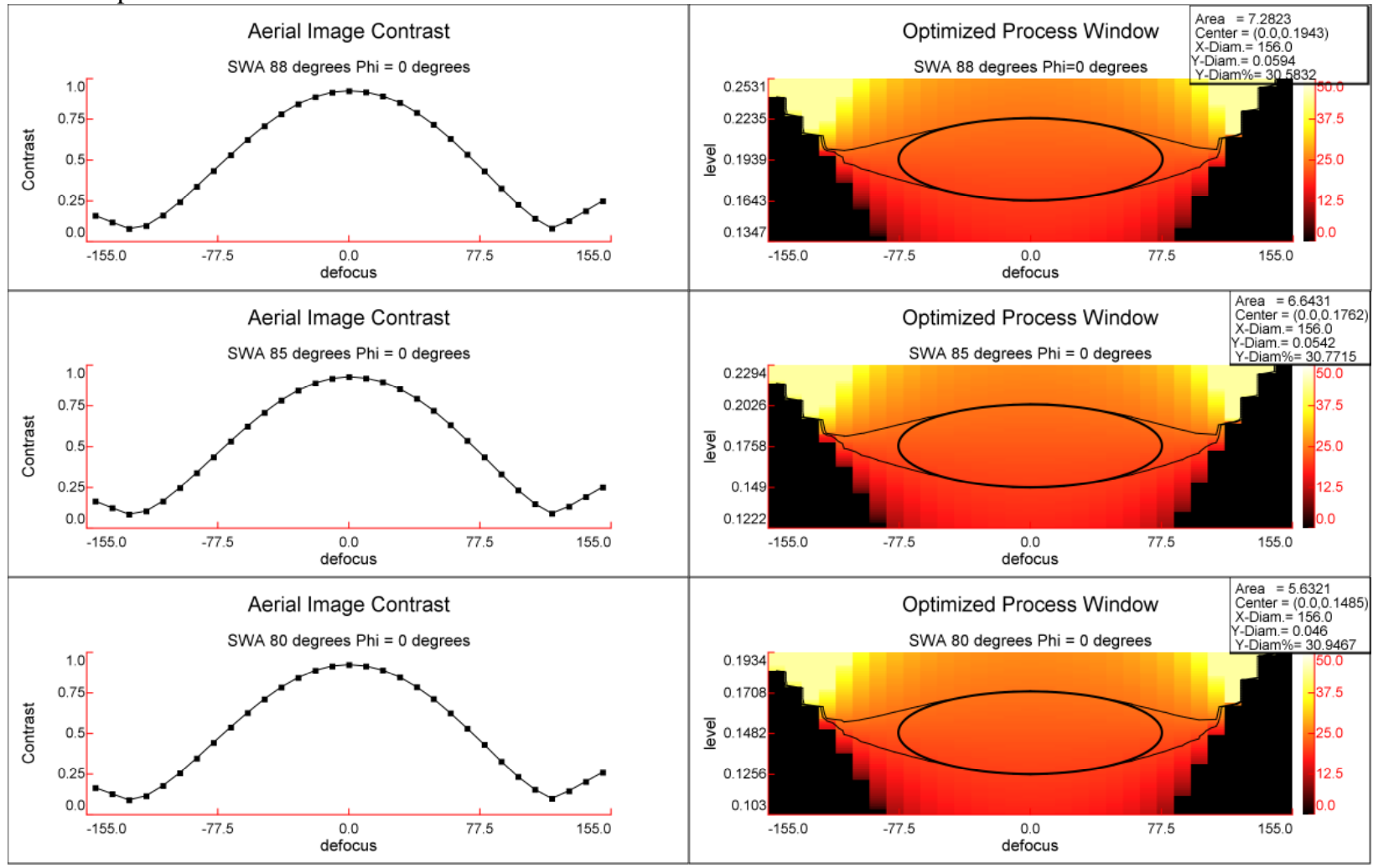

Fig. 2. (left) Contrast through focus curves to determine the range of focus over which the image-log-slope (ILS) delivered a contrast above $50 \%$ for nominal SWA, as varied from $88^{\circ}$ (top), to $85^{\circ}$ (middle), to $80^{\circ}$ (bottom). In each SWA case, the DOF was invariably $\pm 78 \mathrm{~nm}$ for the case of shadowing $\left(\phi=0^{\circ}\right)$.

Fig. 3. (right)Optimized process window for the fixed defocus range of $156 \mathrm{~nm}$ for nominal SWA, as varied from $88^{\circ}$, to $85^{\circ}$, to $80^{\circ}$ for the case of shadowing $\left(\phi=0^{\circ}\right)$. 


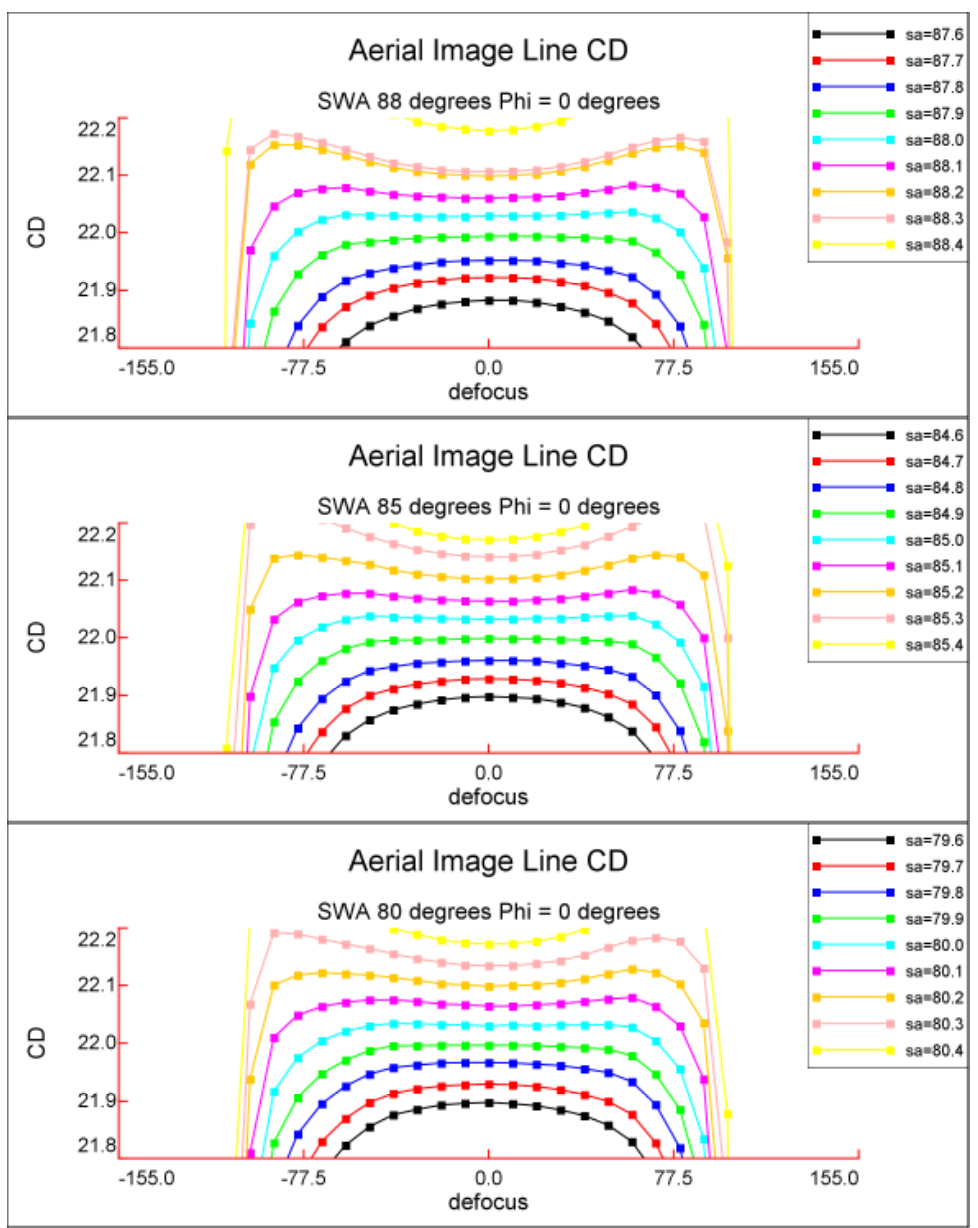

Fig. 4. Sensitivity on changing angle for nominal SWAs of $88^{\circ}$ (top), $85^{\circ}$ (middle), and $80^{\circ}$ (bottom) for the case of shadowing $\left(\phi=0^{\circ}\right.$ ).

We repeated all simulations also for the case that the illumination is parallel to the lines and spaces $\left(\phi=90^{\circ}\right.$ so that no shadowing occurs). Again the nominal angle is found to have little impact on the DOF and sensitivity to small angle perturbations from nominal (see Fig. 5-7).

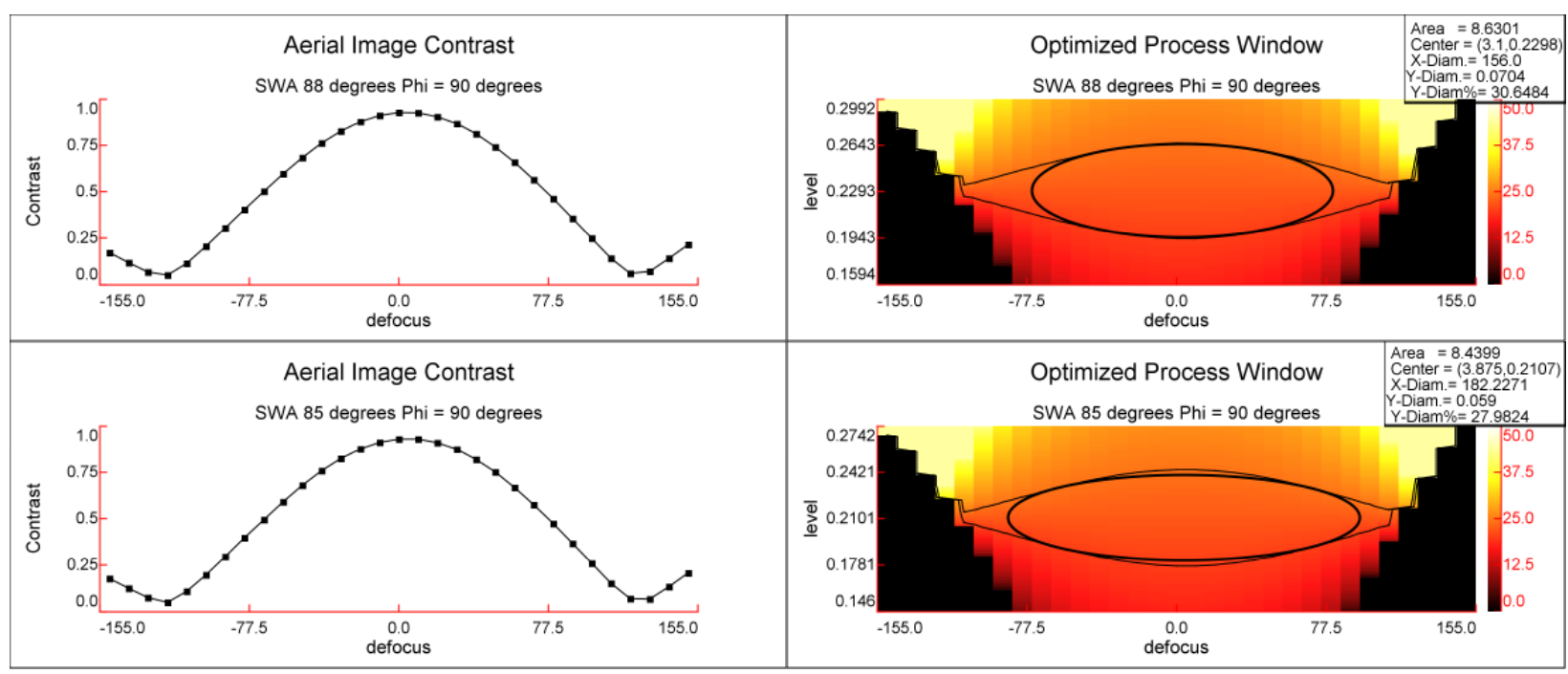




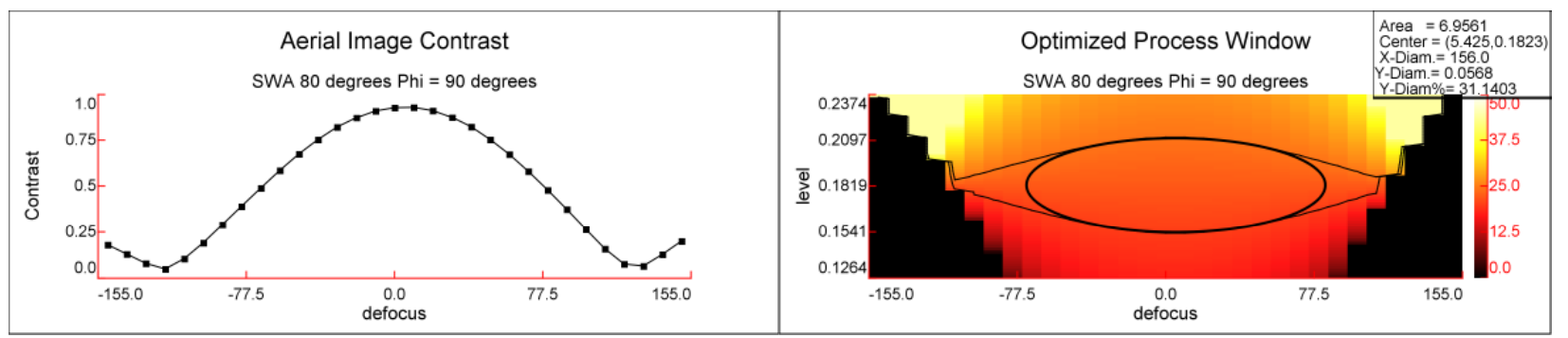

Fig. 5. (left) Contrast through focus curves to determine the range of focus over which the image-log-slope (ILS) delivered a contrast above $50 \%$ for nominal SWA, as varied from $88^{\circ}$ (top), to $85^{\circ}$ (middle), to $80^{\circ}$ (bottom). In each SWA case, the DOF was invariably $\pm 78 \mathrm{~nm}$ for the case of no shadowing $\left(\phi=90^{\circ}\right)$.

Fig. 6. (right)Optimized process window for the fixed defocus range of $156 \mathrm{~nm}$ for nominal SWA, as varied from $88^{\circ}$, to $85^{\circ}$, to $80^{\circ}$ for the case of no shadowing $\left(\phi=90^{\circ}\right)$.

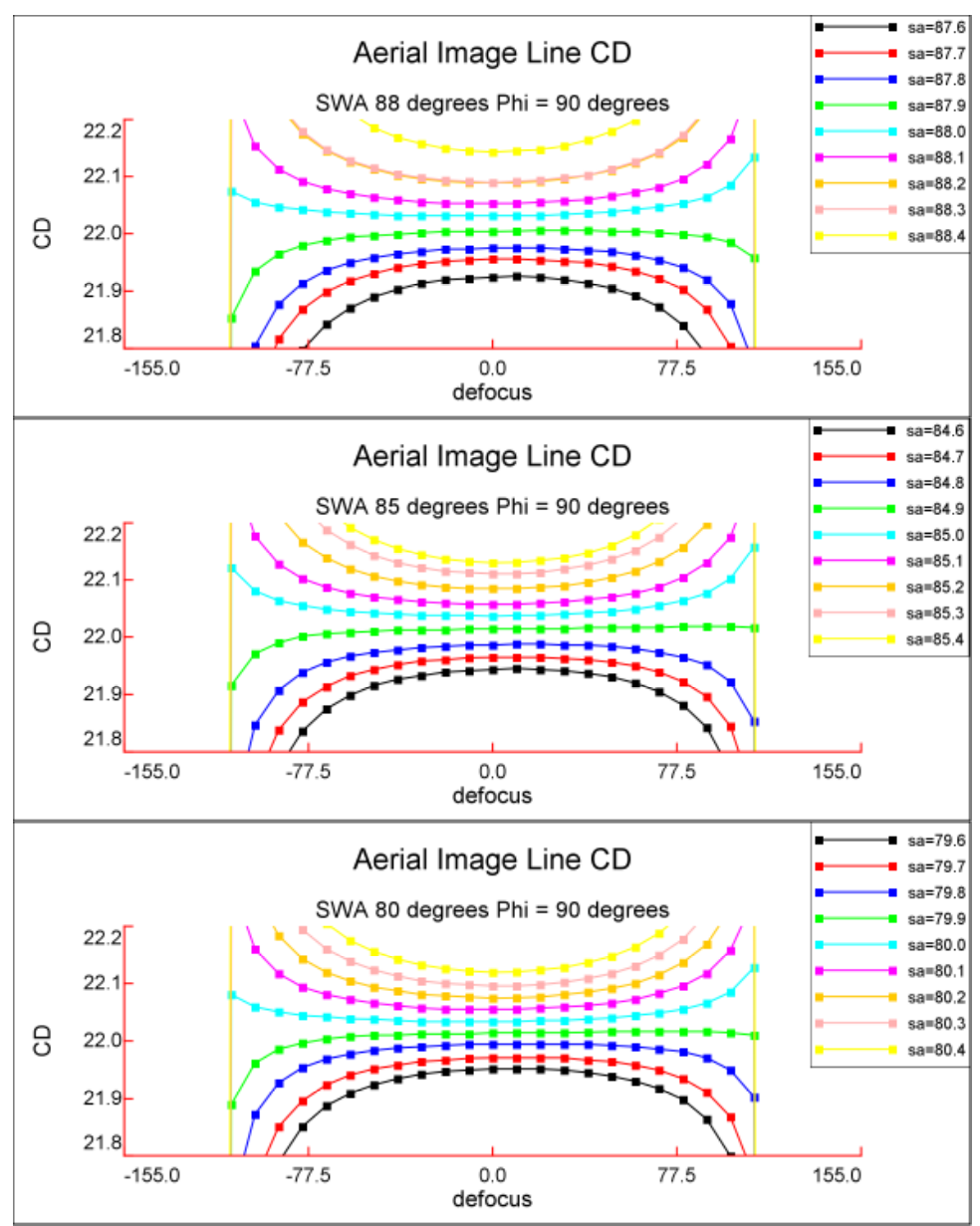

Fig. 7. Sensitivity on changing angle for nominal SWAs of $88^{\circ}$ (top), $85^{\circ}$ (middle), and $80^{\circ}$ (bottom) for the case of no shadowing $\left(\phi=90^{\circ}\right)$.

\section{SHADOW LER AT THE 22-NM HALF-PITCH NODE}

Next, we consider the effect of shadowing on LER transfer from the mask to the wafer, in particular, whether significant changes are introduced between the right and left hand edges. Similar to the simulations above, we use FDTD simulations to obtain a rigorous solution for the field immediately after the patterned mask absorber pattern on a $2.5 \mathrm{~nm}$ Ru capped 40 bilayer ML mirror using EM-Suite[6] by Panoramic technology, with FBC and again a fixed reflectivity 
curve calculated using optical constants [7-8]. The optical system was comprised of an ideal pupil map, NA $=0.32$, $13.4 \mathrm{~nm}$ light at $4^{\circ}$ off-axis, an annular source shape with partial coherence factor of $\sigma=0.35-0.55$, and 50 source points on the half-axis. Again, the patterned TaN mask absorber pattern was 70nm thick. At mask level, the feature was designed to discretely sample various spatial frequencies of LER. For the basic 2D shape of the mask that we exactly replicated through height, we created mask LER by generating a sine wave of amplitude $8 \mathrm{~nm}$ (equivalent to $17 \mathrm{~nm}$ LER at the mask, $4.25 \mathrm{~nm}$ LER at the wafer). The width of the line is fixed at 22-nm in wafer dimensions along the full length. We consider sine waves with wafer dimension spatial frequencies (full period) of 25, 50, 75, 100, 125, 150, 175, and 200nm (see Fig. 8), and illuminated at both $\phi=0^{\circ}$ (perpendicular, shadowing) and $\phi=90^{\circ}$ (parallel, no shadowing). The mask was sampled at $1 \mathrm{~nm} /$ pixel, and the image was resolved to $0.1 \mathrm{~nm} /$ pixel. We assumed a $90^{\circ} \mathrm{SWA}$. Aerial image results were then exported for LER analysis in the offline program SuMMIT [9], and thresholded to size at best focus.
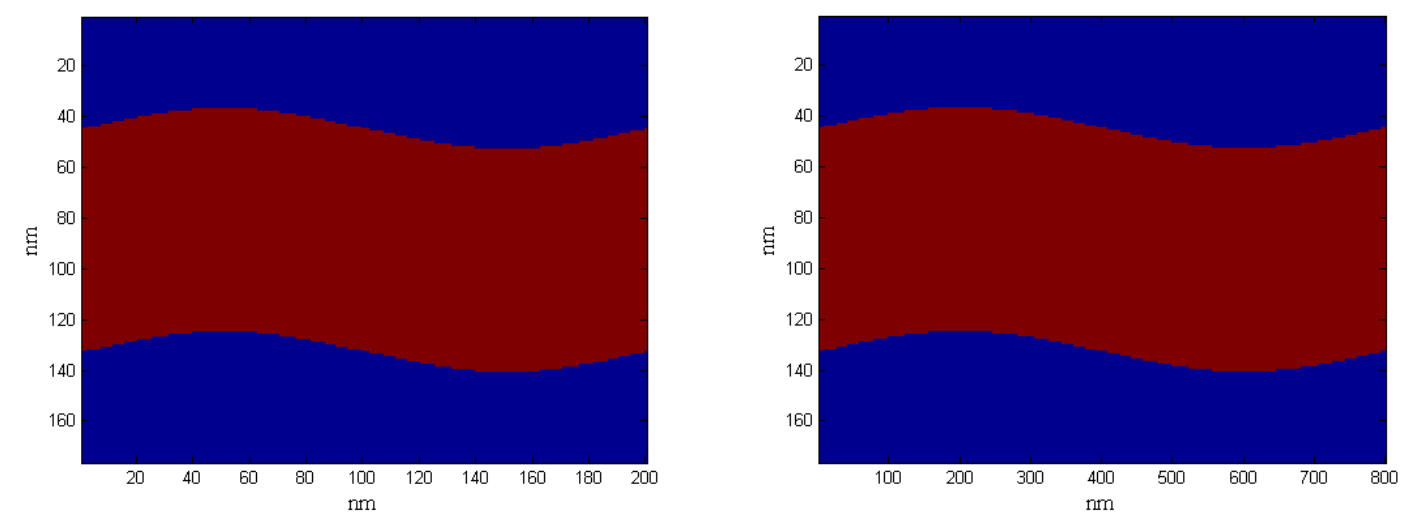

Fig. 8. LER on the mask for full spatial periods of (left) $50 \mathrm{~nm}$ and (right) $200 \mathrm{~nm}$ for $22 \mathrm{~nm}$ features and $\sim 4 \mathrm{~nm}$ LER amplitude (wafer dim. for a $4 \mathrm{X}$ system).

Results show there is not much deviation between the left and right LER due to shadowing (see Fig. 9) (the graph of the $100 \mathrm{~nm}$ full period spatial frequency LER at the mask, ie, $25 \mathrm{~nm}$ at the wafer, was omitted as it was not passed by the optic). For a spatial LER period of $200 \mathrm{~nm}$ at the mask (50nm at the wafer), that is still passed by the optic, at best focus, this amounts to a difference of $0.1 \mathrm{~nm}$ LER between left and right sides of the feature. While a defocus of $160 \mathrm{~nm}$ can exacerbate the difference to at most $0.3 \mathrm{~nm}$ LER, the 'shadow' effect is still not very significant. The effect is even less observable at longer spatial periods, where the left and right LER are nearly identical.

We followed up this study by repeating the data for a mask designed with 2.2nm LER (wafer dimensions) with similar results (see Fig. 10). A plot of the transfer functions for LER of both $\sim 2 \mathrm{~nm}$ and $\sim 4 \mathrm{~nm}$ compared to the contrast of simple 50\% duty cycle lines and spaces is shown in Fig. 11. The latter is what is typically referred to as the contrast transfer function (CTF). As previously demonstrated, the LER transfer function (LTF) is functionally distinct from the CTF [10]. LER transfer functions were based on the non-shadowing case $\left(\phi=90^{\circ}\right)$, and found by dividing the LER actually transferred down to the wafer by the LER initially on the mask (wafer dim.). 


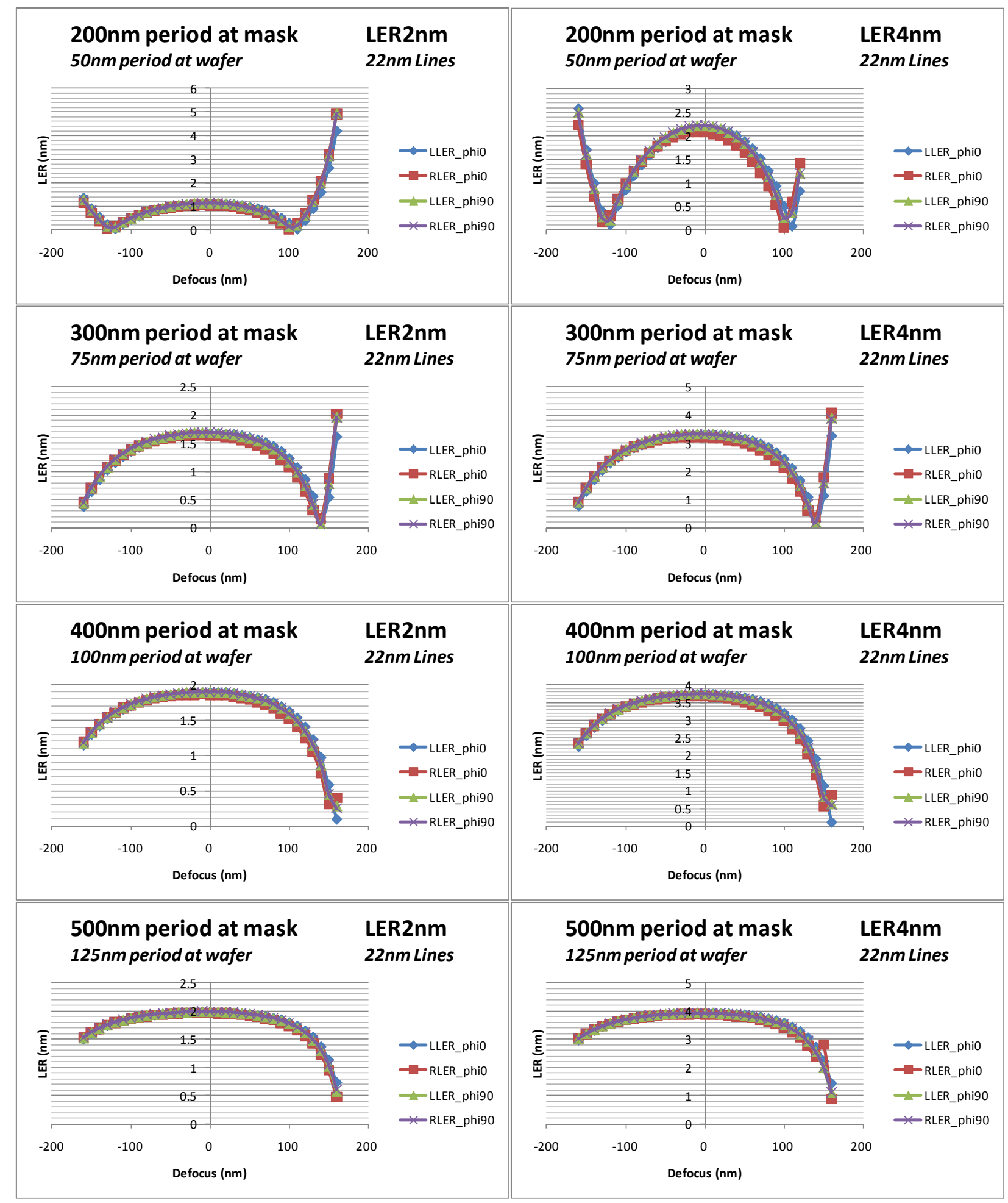




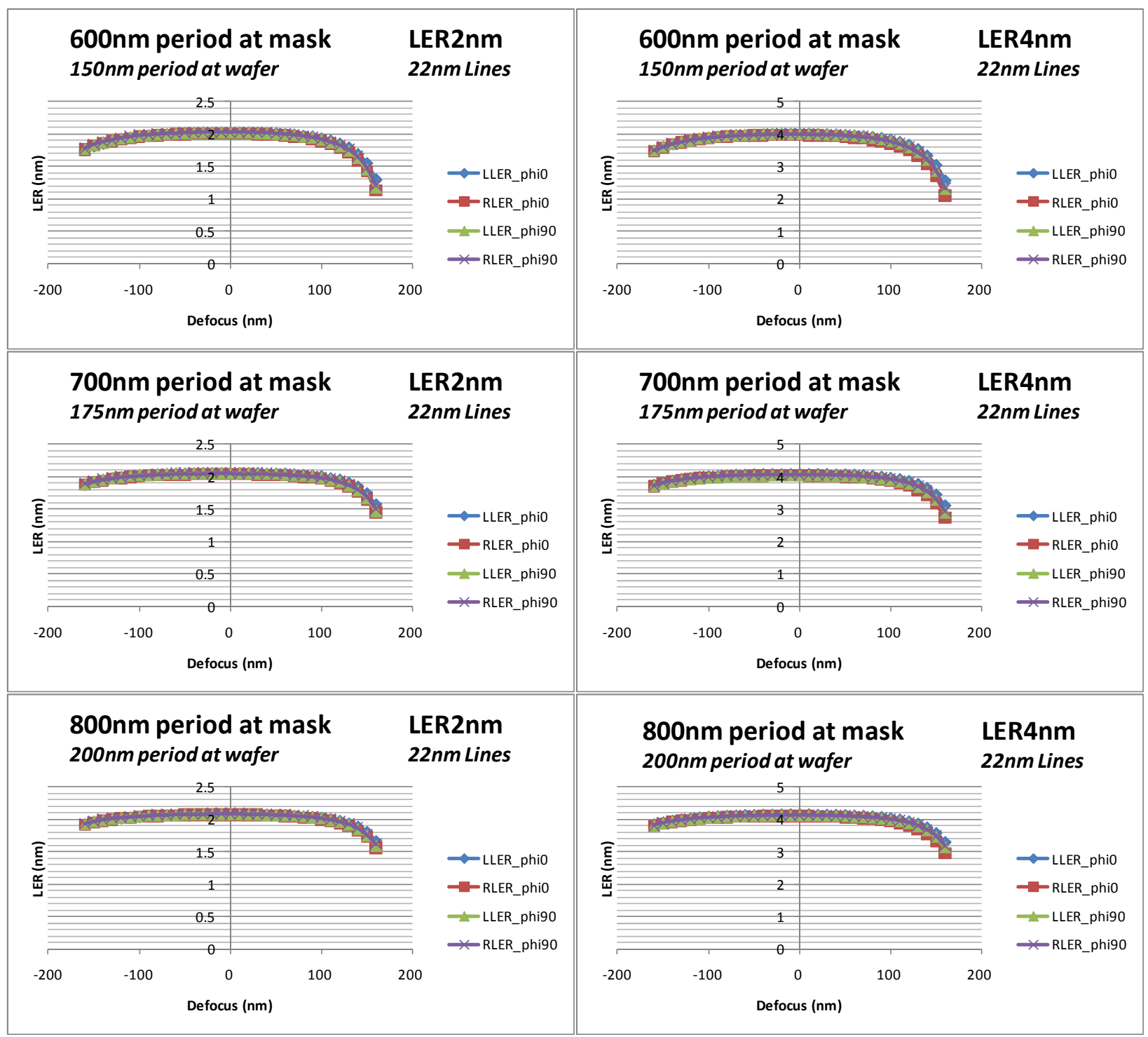

Fig. 9. (left) LER $2 \mathrm{~nm}$, phi $=0,90^{\circ}$, separately for all spatial frequencies, through focus for $22 \mathrm{~nm}$ lines and spaces under disk $\sigma=0.5$ illumination. LLER indicates left edge LER, and RLER indicates right edge LER. Phi $=0^{\circ}$ indicates shadowing, $\mathrm{Phi}=90^{\circ}$ indicates no shadowing. The graph for LER Spatial frequency of full period $100 \mathrm{~nm}$ at the mask was omitted as it was not passed by the optical system.

Fig. 10. (right) LER $4 \mathrm{~nm}$, phi $=0,90^{\circ}$, separately for all spatial frequencies, through focus for $22 \mathrm{~nm}$ lines and spaces under disk $\sigma=$ 0.5 illumination. LLER indicates left edge LER, and RLER indicates right edge LER. Phi $=0^{\circ}$ indicates shadowing, Phi $=90^{\circ}$ indicates no shadowing. The graph for LER Spatial frequency of full period 100nm at the mask was omitted as it was not passed by the optical system. 


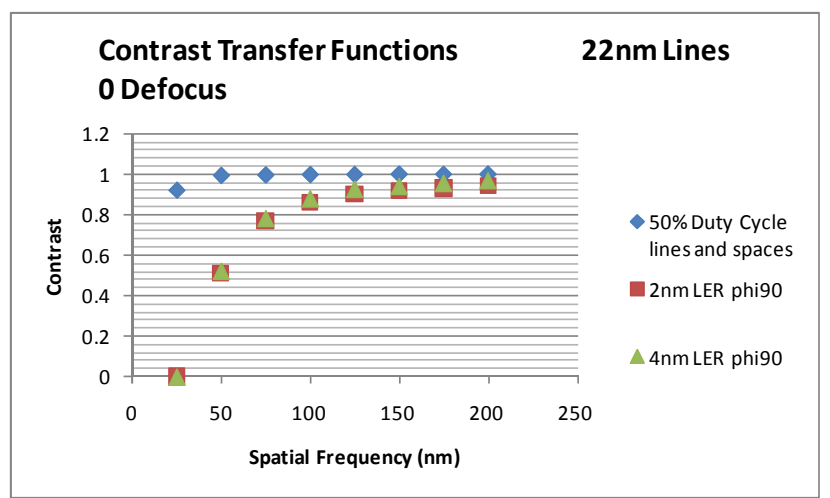

Fig. 11. A plot of the transfer functions for LER of both $2 \mathrm{~nm}$ and $4 \mathrm{~nm}$ compared to the contrast of simple $50 \%$ duty cycle lines and spaces (what can be considered the traditional MTF).

\section{SHADOW LER AT THE 16-NM HALF-PITCH NODE}

We expanded our study to consider what happens if the $\mathrm{k}_{1}$-factor of the optic is stressed. In particular, 16nm lines and spaces on an NA $=0.32$ optic with crosspole illumination with $\sigma=0.20$ and displaced $\mathrm{dx}=0$, $\mathrm{dy}=0.67$ (pole position optimized for $16 \mathrm{~nm}$ lines and spaces), this time with off-axis illumination at $6^{\circ}$, all other settings the same. We did this for LER amplitudes of 1.1 and $2.2 \mathrm{~nm}$ (wafer dimensions).

Results for this case show a strong difference between left-side and right-side LER (see Fig. 12 \& 13). The strongest difference is seen at the smallest spatial frequency period of the LER mask sine wave that passes both the shadowing and non-shadowing LER: $200 \mathrm{~nm}$ at the mask (50nm at the wafer). At zero defocus, the difference is about $0.2 \mathrm{~nm}$ LER for an initial LER of $2 \mathrm{~nm}$ (wafer dimensions). As far out as $-160 \mathrm{~nm}$, the difference is as great as $0.4 \mathrm{~nm}$ LER. As the full period of the sine wave LER on the mask increases to $800 \mathrm{~nm}$ (200nm at the wafer), the difference between left and right LER lessens significantly. The LER transfer functions for the $16 \mathrm{~nm}$ lines and spaces were based again on the nonshadowing case $\left(\phi=90^{\circ}\right)$, and reconfirm that the system remains linear between LER amplitudes of $\sim 1 \mathrm{~nm}$ to $\sim 2 \mathrm{~nm}$ (see Fig. 14). It is interesting to note that the smallest spatial frequency period of LER on the mask, $100 \mathrm{~nm}$ (or $25 \mathrm{~nm}$ wafer dim.), passed only in the shadowing case $\left(\phi=0^{\circ}\right)$, with an increase in the LER as the image shifts through focus.

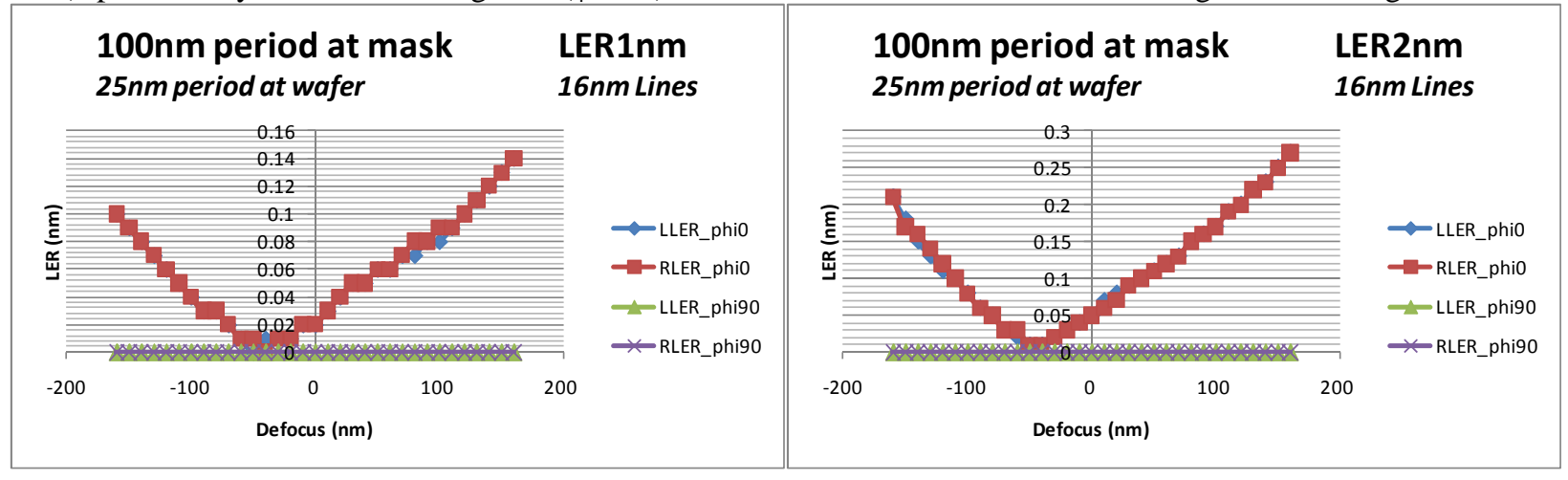




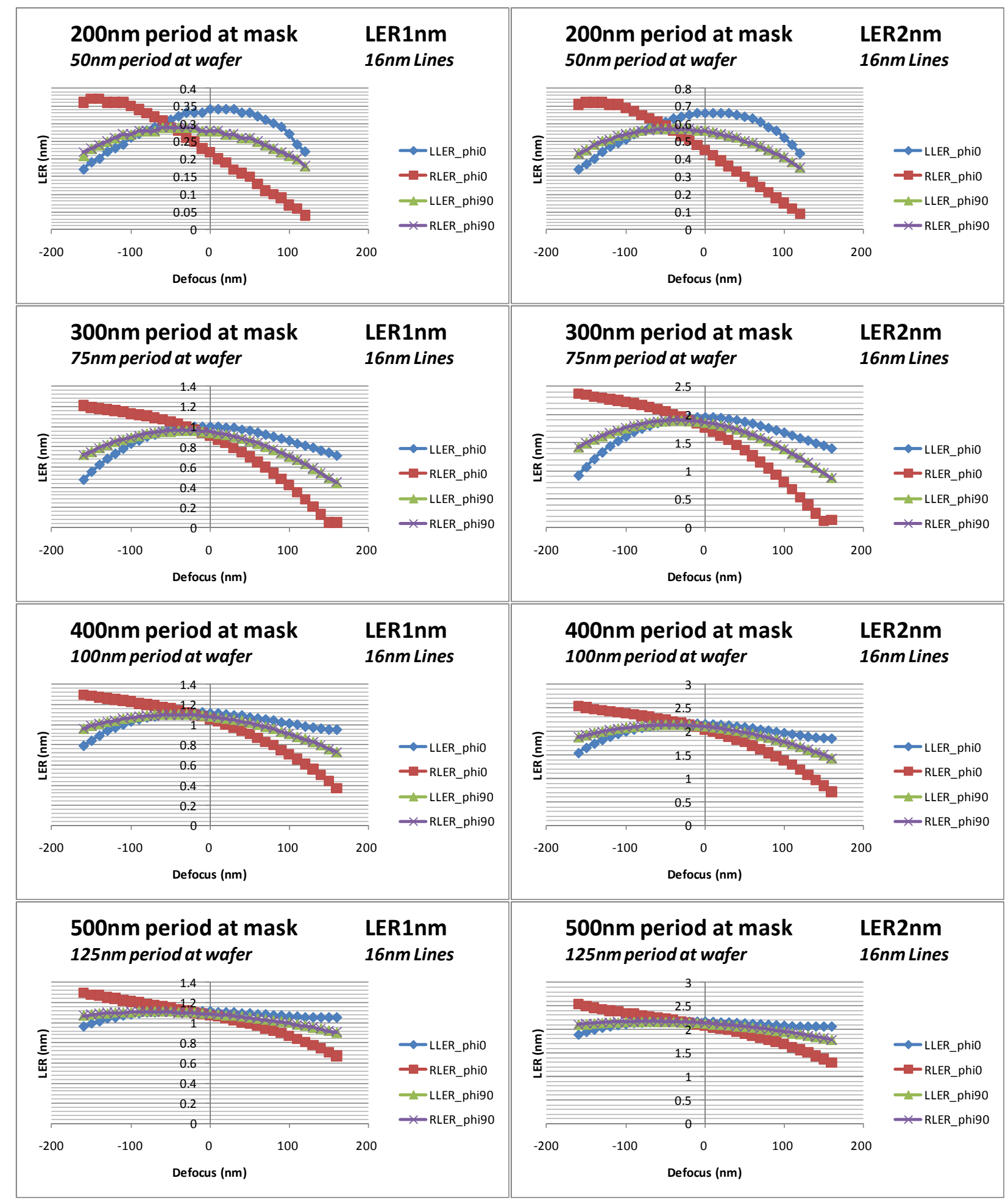




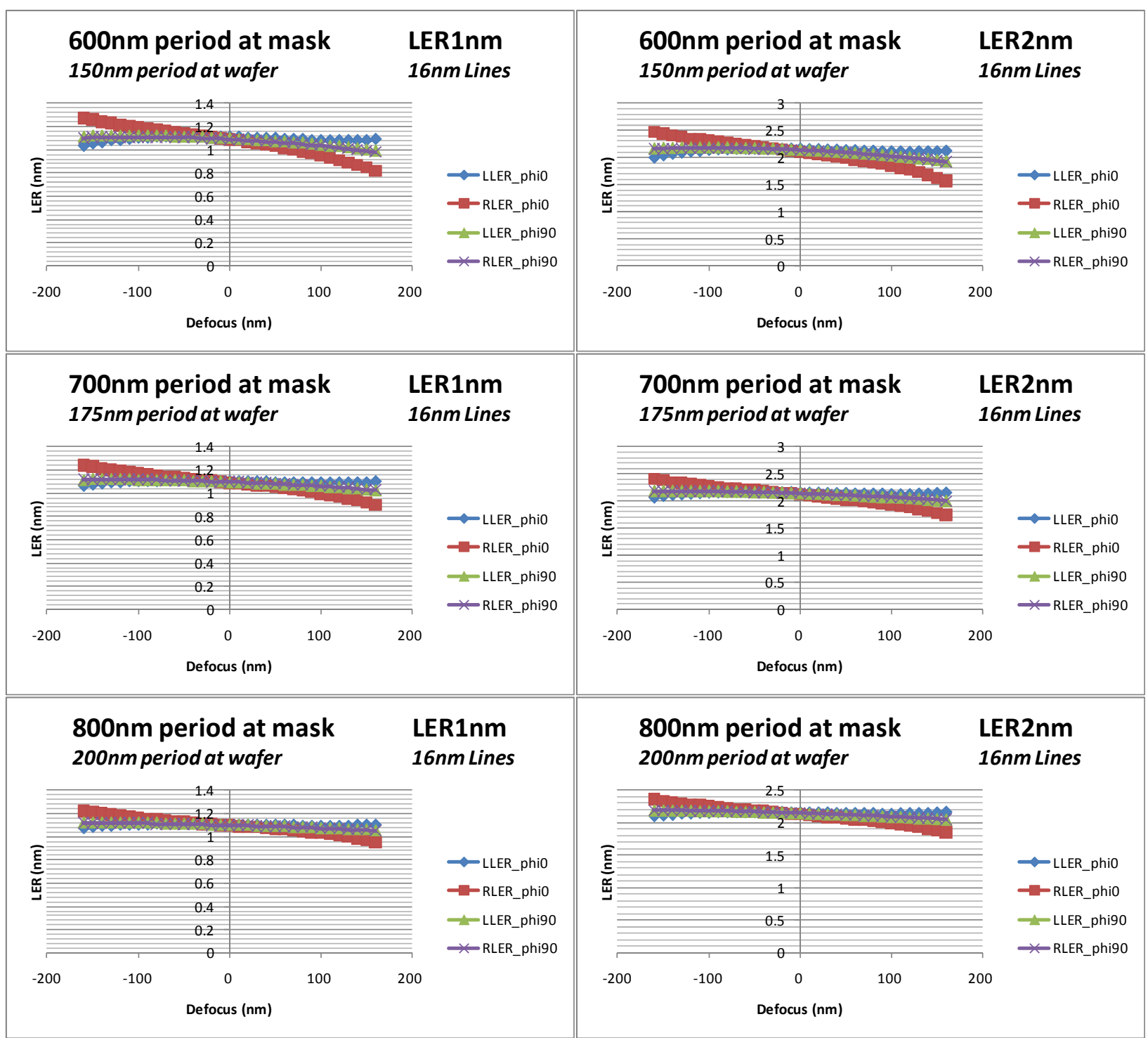

Fig. 12. (left) LER $1 \mathrm{~nm}$, phi $=0,90^{\circ}$, separately for all spatial frequencies, through focus for $16 \mathrm{~nm}$ lines and spaces under crosspole $\sigma$ $=0.2$ illumination $(\mathrm{dx}=0, \mathrm{dy}=0.67)$. LLER indicates left edge LER, and RLER indicates right edge LER. Phi $=0^{\circ}$ indicates shadowing, $\mathrm{Phi}=90^{\circ}$ indicates no shadowing.

Fig. 13. (right) LER $2 \mathrm{~nm}$, phi $=0,90^{\circ}$, separately for all spatial frequencies, through focus for $16 \mathrm{~nm}$ lines and spaces under crosspole $\sigma$ $=0.2$ illumination $(\mathrm{dx}=0, \mathrm{dy}=0.67)$. LLER indicates left edge LER, and RLER indicates right edge LER. Phi $=0^{\circ}$ indicates shadowing, $\mathrm{Phi}=90^{\circ}$ indicates no shadowing. 


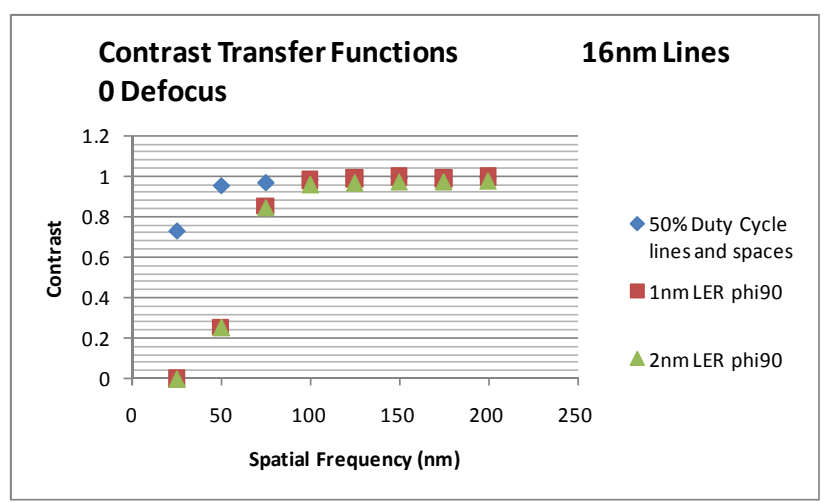

Fig. 14. A plot of the transfer functions for LER of both $2 \mathrm{~nm}$ and $4 \mathrm{~nm}$ compared to the contrast of simple $50 \%$ duty cycle lines and spaces (what can be considered the traditional MTF).

\section{SUMMARY}

In the course of our investigation of SWA, we found that the nominal angle has no effect on process window size or sensitivity to SWA deviations from nominal. For nominal $80^{\circ}, 85^{\circ}, 88^{\circ}$ SWA, the amount of angle tolerance for $0.2 \mathrm{~nm}$ change in $\mathrm{CD}$ was in total $0.5^{\circ}$.

For "shadow" LER, we found minimal difference between left and right LER for the 22nm half-pitch node under disk $\sigma$ $=0.5$ illumination for absorber thickness of $70 \mathrm{~nm}$, to at the most $0.1 \mathrm{~nm}$ LER at best focus. For $16 \mathrm{~nm}$ half-pitch under crosspole $\sigma=0.2$ (pole position optimized for $16 \mathrm{~nm}$ lines and spaces), however, there is a significant difference between left and right LER transfer resulting from the shadowing.

The authors are grateful for the support of this work by the National Science Foundation EUV Science and Technology Center. The work was performed at Lawrence Berkeley National Laboratory's Advanced Light Source synchrotron facility and was supported in part by SEMATECH through the U.S. Department of Energy under Contract No. DEAC02-05CH11231.

\section{REFERENCES}

[1] M. Besacier, and P. Schiavone, "Shadowing effect minimization in EUV mask by modeling", Proc. SPIE 5446, (2004) 849.

[2] M. Yoo, Y. Jeon, H. Oh, and J. Ahn, "Reduction of the absorber shadow effect by changing the absorber side wall angle in extreme ultraviolet lithography," J. Korean Phys. Soc. 46, 1020 (2005).

[3] M. Sugawara, and I. Nishiyama, "Impact of Slanted Absorber Side Walls on Critical Dimension Error in Extreme Ultraviolet Lithography”, Jpn. J. Appl. Phys. 46, 84 (2007).

[4] M. Sugawara, and I. Nishiyama, and M. Takai, "Effect of mask pattern correction for off-axis incident light in extreme ultraviolet lithography”, J. Vac. Sci. Technol. B 22, (2004) 3053.

[5] M. Sugawara, A.Chiba, and I. Nishiyama, "Effect of incident angle of off-axis illumination on pattern printability in extreme ultraviolet lithography", J. Vac. Sci. Technol. B 21, (2003) 2701.

[6] Panoramic Technology, Inc., "EM-suite/Hyperlith” (2009), see http://www.panoramictech.com.

[7] Gullikson, E., "Multilayer reflectivity," (2007).

[8] Palik, E. D. and Ghosh, G., Handbook of Optical Constants of Solids, Academic Press, San Diego, CA (1997). http://henke.lbl.gov/optical_constants. 
[9] EUV Technology Corp., http://www.euvl.com/summit.

[10] P. Naulleau and G. Gallatin, "Line-edge roughness transfer function and its application to determining mask effects in EUV resist characterization," Appl. Opt. 42, 3390-3397 (2003).

\section{DISCLAIMER}

This document was prepared as an account of work sponsored by the United States Government. While this document is believed to contain correct information, neither the United States Government nor any agency thereof, nor The Regents of the University of California, nor any of their employees, makes any warranty, express or implied, or assumes any legal responsibility for the accuracy, completeness, or usefulness of any information, apparatus, product, or process disclosed, or represents that its use would not infringe privately owned rights. Reference herein to any specific commercial product, process, or service by its trade name, trademark, manufacturer, or otherwise, does not necessarily constitute or imply its endorsement, recommendation, or favoring by the United States Government or any agency thereof, or The Regents of the University of California. The views and opinions of authors expressed herein do not necessarily state or reflect those of the United States Government or any agency thereof or The Regents of the University of California. 